\title{
As especificidades do sistema de inovação do setor saúde ${ }^{1}$
}

\author{
The specificities of the health sector innovation system
}

\section{EDUARDO DA MOTTA E ALBUQUERQUE² JOSÉ EDUARDO CASSIOLATO ${ }^{3}$}

\begin{abstract}
RESUMO: Este artigo discute o sistema de inovação biomédica. Esse sistema setorial é uma interseção entre o sistema de inovação e as instituições de assistência social. A dinâmica inovadora no setor da saúde possui várias características distintas, especialmente a importância das universidades e a pesquisa acadêmica para as inovações biomédicas. A especificidade do cuidado em saúde como atividade econômica explica o papel das instituições nesse setor. As instituições e a regulamentação moldam a direção do progresso tecnológico, influenciando o desempenho econômico, industrial e social de todo o setor da saúde.

PALAVRAS-CHAVE: Economia da saúde; sistemas de inovação; sistemas de bem-estar social.

ABSTRACT: This paper discusses the biomedical innovation system. This sectoral system is an intersection between the innovation system and the welfare institutions. The innovative dynamics in the health sector has various distinctive characteristics, specially the importance of the universities and academic research for the biomedical innovations. The specificity of health care as an economic activity explains the role of institutions in this sector. Institutions and regulation shape the direction of technological progress, influencing the economic, industrial and social performance of the whole health sector.
\end{abstract}

KEYWORDS: Health economics; innovation systems; social welfare systems. JEL Classification: I10, O30, H75.

\footnotetext{
${ }^{1}$ Este artigo sintetiza os principais pontos de uma pesquisa realizada por solicitação da Federação das Sociedades de Biologia Experimental (FeSBE). Este trabalho contou com a participação de Aletheia Zanow, Márcia Rapini, Regina Fernandes (FACE-UFMG) e de Alexandre Diniz (Medicina-UFMG). As sugestões de dois pareceristas anônimos da Revista de Economia Política foram importantes para aperfeiçoar o texto. Os erros são responsabilidade exclusiva dos autores.

${ }^{2}$ Centro de Desenvolvimento e Planejamento Regional da Universidade Federal de Minas Gerais CEDEPLAR-UFMG, Belo Horizonte/MG, Brasil. E-mail: albuquerque@cedeplar.ufmg.br; Orcid: 00000002-1591-875X.

${ }^{3}$ Instituto de Economia da Universidade Federal do Rio de Janeiro (IE-UFRJ), e-mail: cassio@ie.ufrj.br; Orcid: 0000-0001-8377-6012.
} 


\section{INTRODUÇÃO}

As especificidades do progresso científico-tecnológico no setor saúde devem ser cuidadosamente investigadas, dados o enorme impacto positivo desse progresso sobre o bem-estar social e sobre o desenvolvimento econômico. A literatura econômica sobre o tema tem sido pouco explorada nas discussões realizadas no Brasil.

O objetivo deste artigo é apresentar as principais características do sistema de inovação no setor saúde, a partir da avaliação da estrutura institucional construída em países capitalistas desenvolvidos.

O conceito de sistema nacional de inovação é utilizado como fio-condutor deste artigo. A utilidade desse conceito reside no fato de o mesmo tratar explicitamente questões importantes, ignoradas em modelos mais antigos de mudança tecnológica - especificamente o da diversidade e do papel dos investimentos intangíveis em atividades de aprendizado inovativo. Tal noção envolve, portanto, não apenas empresas, mas também, instituições de ensino e pesquisa, de financiamento, governo etc. Além disso - e baseando-se na consideração que uma diversidade significativa existe entre os países e instituições na forma, nível e padrão dos investimentos em aprendizado - focalizam-se particularmente as ligações entre instituições e suas estruturas de incentivos e capacitações.

É possível desagregar um sistema nacional de inovação em diferentes setores, pois as características do progresso tecnológico e dos fluxos de informações científico-tecnológicas variam consideravelmente (Pavitt, 1984; Breschi \& Malerba, 1997). Como o setor saúde pode ser demarcado de outras atividades econômicas em termos da dinâmica inovativa, é razoável discutir a idéia de um sub-sistema de inovação no setor saúde (Hicks \& Katz, 1996). Porém, deve-se ter em mente que a visão "setorial" evolucionista aqui apresentada difere significativamente da perspectiva setorial tradicional, pois esta não captura situações onde as fronteiras dos "setores" encontram-se em mutação, tornando-se fluidas (Mytelka \& Delapierre, 1999). Assim, de uma perspectiva dinâmica, os "setores" devem ser reconceitualizados enquanto sistemas mais amplos baseados em "clusters" de tecnologias e soluções. É sob tal perspectiva que o sistema de inovação de saúde será aqui analisado.

A seção I deste artigo investiga a especificidade do sistema de inovação do setor saúde, buscando identificar os fluxos de informação científica e tecnológica que compõem as complexas interações ligadas à problemática da saúde. A seção II discute as interações recíprocas entre os sistemas de inovação e de bem-estar, uma outra importante especificidade na medida que o progresso tecnológico no setor tem implicações diretas sobre a ampliação do bem-estar individual e social. A seção III conclui o trabalho. 


\section{O SISTEMA DE INOVAÇÃO DO SETOR SAÚDE EM PAÍSESDESENVOLVIDOS}

\section{I.1. Características Gerais dos Fluxos de Informação Científica e Tecnológicos no Setor Saúde}

Três pontos de partida já desenvolvidos na literatura podem ser utilizados para introduzir essa discussão.

Em primeiro lugar, é interessante recuperar uma elaboração proposta por estudiosos do setor saúde: o complexo médico-industrial (Cordeiro, 1980, p. 113). Trata-se de uma articulação que envolve a assistência médica, as redes de formação profissional (escolas, universidades), a indústria farmacêutica, a indústria produtora de equipamentos médicos e instrumentos de diagnóstico.

Em segundo lugar, a sugestão de Hicks \& Katz (1996) da existência de um sistema biomédico de inovação. A partir de um estudo sobre as contribuições dos hospitais para a produção científica britânica, Hicks \& Katz (1996, p. 304) encontraram tantas singularidades que sugeriram a possibilidade de existência de um sistema biomédico de inovação, no qual os hospitais participariam intensamente.

Em terceiro lugar, o trabalho editado por Gelijns \& Rosenberg (1995) detalhou várias facetas da interação entre as universidades e a indústria na geração de tecnologia médica. Estudos desse livro também apontam inúmeras particularidades na interação produtor-usuário, na qual a profissão médica desempenha importante papel no desenvolvimento de inovações, assim como em seu aperfeiçoamento.

De uma forma exploratória e preliminar, algumas características gerais do sistema de inovação do setor saúde podem ser apontadas:

1. O papel das universidades e instituições de pesquisa: o número de fluxos deinformação científica e tecnológica que se originam ou que se destinam para essas instituições é grande. As universidades se caracterizariam como um verdadeiro foco e centro de convergência de fluxos. Essa posição crucial é uma manifestação da proximidade que o progresso tecnológico do setor tem com a ciência.

2. A assistência médica, envolvendo hospitais, clínicas, postos médicos etc.,participa também intensamente dos fluxos, interagindo fortemente com as indústrias do setor e com a universidade. Hospitais e centros médicos acadêmicos apresentam demandas para os componentes do sub-sistema $\mathrm{e}$ interagem ao longo do seu desenvolvimento.

3. As instituições de regulação (como o Food and Drugs Administration FDAnorte-americano) e associações profissionais e escolas médicas cumprem um papel de filtro das inovações geradas pelas universidades e indústrias. Essa combinação de instituições aponta o papel singular desempenhado por ambientes seletivos nãomercantis no setor saúde. Aliás, 
Nelson \& Winter (1982, pp. 268-272) já haviam chamado atenção para o papel da profissão médica na seleção de novos tratamentos.

4. As firmas do setor têm graus diferentes de interação com as universidades.

5. A saúde pública entra com um papel importante, tendo interações diretascom as universidades e instituições de pesquisa, além de receber as inovações provenientes do complexo médico-industrial. As interações processam-se de forma mediada pelo sistema de assistência médica.

6. A efetividade das inovações, implementadas pela assistência médica e saúdepública, repercute diretamente sobre o bem-estar da população, que é o objetivo final do conjunto do sub-sistema de inovação da saúde. Indicadores das melhorias de bem-estar social são a ampliação da expectativa de vida (World Bank, 1993) e a redução de AVAIs (anos de vida ajustados por invalidez) (Murray \& Lopez, 1996).

\section{I.2. Um Sistema Setorial Fortemente Baseado na Ciência}

A literatura da Economia da Tecnologia enfatiza as diferenças inter-setoriais nas relações entre ciência, tecnologia e produção. Freeman \& Soete (1997), por exemplo, discorrem longamente sobre a emergência das tecnologias relacionadas à ciência, Pavitt (1984) constrói uma taxonomia de fluxos tecnológicos na qual um setor "baseado na ciência" é destacado. É enfatizada por acadêmicos da Economia da Tecnologia a proximidade e o entrelaçamento existente entre a ciência e a tecnologia no setor saúde (Nelson, 1995; Gelijns \& Rosenberg, 1995).

Gelijns \& Rosenberg (1995, p. 5) destacam duas características. Em primeiro lugar, a inovação médica é crescentemente dependente de pesquisas interdisciplinares. Em medicamentos, por exemplo, uma nova droga requer o trabalho de químicos, biólogos moleculares, imunologistas, engenheiros químicos, clínicos etc. $\mathrm{Na}$ indústria de equipamentos médicos, por sua vez, inovações requerem o trabalho de físicos, engenheiros eletrônicos, especialistas em novos materiais, especialistas médicos etc.

Ou seja, a produção de inovações no setor saúde tem por pré-requisito uma estrutura de formação universitária e de pós-graduação abrangente e razoavelmente sofisticada, dado o tipo de interação e interdisciplinaridade que ela apresenta. Em segundo lugar, segundo Gelijns \& Rosenberg (1995) a inovação médica depende pesadamente das interações entre universidades (especialmente centros médicos acadêmicos) e empresas industriais.

Nelson (1995), por sua vez, confessou-se impressionado pela relevância do entrelaçamento entre ciência e tecnologia para a pesquisa e a inovação médica. Destacou que o advento da biotecnologia moderna certamente tem exacerbado essa relação. Essa observação de Nelson fundamenta a existência de fluxos de informação fortes nos dois sentidos entre as universidades e as indústrias biotecnológicas.

$\mathrm{Na}$ verdade, talvez existam relações mais complexas. É possível encontrar na 
literatura exemplos de verdadeira "inversão" nos papéis tradicionais das universidades e indústrias na geração de conhecimentos públicos e conhecimentos apropriados privadamente. Rosenberg \& Nelson (1995, p. 339) estudaram patentes de universidades norte-americanas, analisando o peso relativo das patentes universitárias sobre o total de patentes do setor e ordenando os setores industriais e tecnológicos nesse sentido. O resultado encontrado mostra que os cinco setores líderes são da área biomédica. ${ }^{4}$

A pesquisa relatada em Klevorick et alli (1995) aponta que a produção científica universitária foi considerada de alta relevância para as indústrias de equipamentos médicos, medicamentos e biotecnologia. Biologia foi considerada de alta relevância para a indústria de medicamentos (além de alimentação animal, processamento de frutas e vegetais); Química para medicamentos (além de alimentação animal e carnes) e Ciências Médicas para instrumentos médicos e cirúrgicos e medicamentos (além da indústria de café).

Rosenberg \& Nelson (1994), avaliando esses resultados, discutem as influências indiretas de alguns setores científicos: os avanços mais fundamentais em física e matemática seriam traduzidos para a indústria através de disciplinas aplicadas (como química, engenharias etc). Esse comentário é importante porque o padrão de desenvolvimento tecnológico do setor de equipamentos médicos e cirúrgicos é profundamente dependente desse tipo indireto de relações.

Por sua vez, Mansfield (1991) entrevistou 66 grandes empresas norte-americanas, em sete indústrias. Os resultados apontam a indústria farmacêutica como a mais fortemente dependente de pesquisas acadêmicas: a) $27 \%$ dos novos produtos e $22 \%$ dos novos processos não teriam sido desenvolvidos na ausência de pesquisas acadêmicas recentes; b) $17 \%$ dos novos produtos e $8 \%$ dos novos processos foram desenvolvidos com ajuda substancial de pesquisas acadêmicas recentes.

Finalmente, Narin, Hamilton \& Olivastro (1997) em um estudo encomendado pela National Science Foundation sobre a dependência da indústria norte-americana em relação à ciência financiada com recursos públicos (NSF, 1998, p. 6-20), pesquisaram as referências existentes nas patentes a trabalhos científicos. Comparando os dados de 1987-1988 e 1993-1994, tais autores identificaram um crescimento de $30 \%$ no total de patentes. Para o mesmo período o total de referências a publicações científicas com autores norte-americanos passou de 17.000 para 50.000 (aumento de quase 200\%). Narin et alli (1997) demonstram as articulações crescentes entre as inovações da indústria dos Estados Unidos e as pesquisas da comunidade acadêmica.

Tais análises indicam a importância da ciência financiada publicamente para o dinamismo tecnológico da indústria norte-americana. Desagregando os setores envolvidos, os resultados de Narin et alli indicam que as patentes relacionadas a

\footnotetext{
${ }^{4}$ Os cinco setores líderes na patenteação universitária são: 1) engenharia genética, 2) química orgânica (proteínas), 3) biologia molecular e microbiologia, 4) cirurgia e 5) compostos orgânicos.
} 
drogas e medicamentos são as que apresentam a mais forte dependência em relação à ciência pública.

No geral, os artigos científicos citados pelas patentes das indústrias norte-americanas têm uma forte origem em instituições públicas $(43,9 \%$ dos Estados Unidos; $29,4 \%$ estrangeiras), sendo que a soma das patentes de drogas e medicamentos apresentam a maior participação total de instituições públicas $(79,1 \%$ de todas as citações).

Uma observação final, útil tanto para afirmar o peso das atividades científicas no setor saúde como para identificar outra originalidade de suas fontes em relação a outros setores econômicos, é apresentada pelo trabalho de Hicks \& Katz (1996): o papel dos hospitais como parte do sistema de pesquisa.

Hicks \& Katz (1996) investigaram os hospitais britânicos e suas relações com outros institutos e universidades. Através da análise de colaborações entre autores de diferentes instituições, tais autores encontraram que os hospitais são fortemente articulados com institutos da Special Health Authority (SHA) e do sistema de British Post-Graduate Medical Research (BPG), formando um grupo de vínculos de pesquisa fortes, em oposição ao grupo constituído por indústrias, governo e universidades. A participação dos hospitais britânicos, somados aos institutos da SHA/BPG foram responsáveis por $25 \%$ da produção científica britânica nos anos oitenta (p. 304). Um resultado expressivo e pouco estudado, destacam.

\section{I.3. O Financiamento da Pesquisa Biomédica}

O peso do setor público na geração de conhecimentos indispensáveis ao progresso tecnológico do setor foi apontado de diversas maneiras no tópico anterior. Bond \& Glynn (1995) sistematizam informações sobre os recursos e as fontes de financiamento do P\&D biomédico. Em 1993 os Estados Unidos gastaram US\$ 30 bilhões com o P\&D no setor. Nesse ano, o gasto com P\&D nos Estados Unidos alcançou o total de US\$160,98 bilhões (NSF, 1998, p. A-124). Esses recursos provieram das seguintes fontes: a) indústria (setor privado): $50 \%$; b) governo federal: $39 \%$; c) governos estaduais e municipais: $7 \%$; d) entidades não-lucrativas: $4 \%$.

A participação do governo federal nesses gastos cresceu de forma intensa desde 1940. Naquele ano, segundo Bond \& Glynn (p. 17), as corporações norte-americanas investiram US\$25 milhões, as entidades não-lucrativas US\$ 17 milhões e o governo federal apenas US\$ 3 milhões (7\% do total, valores em dólares correntes). O crescimento da participação do governo federal nas pesquisas biomédicas não se deu de forma isolada, sendo representativa do crescimento do envolvimento do apoio público à pesquisa básica que ocorreu em vários setores científicos (ver Rosenberg \& Nelson, 1994).

Os dados apresentados por Bond \& Glynn permitem cinco observações.

Em primeiro lugar, o caráter altamente intensivo em $P \& D$ da indústria farmacêutica. Em 1993, a indústria farmacêutica e a biotecnológica aplicaram em P\&D $17 \%$ do total das vendas, intensidade de P\&D que era aproximadamente o dobro das outras indústrias de alta tecnologia (Bond \& Glynn, pp. 25-26). 
Em segundo lugar, o quadro diferente do P\&D da indústria de instrumentos médicos, que conta com uma pequena participação no orçamento federal. Uma explicação é a elevada dependência das inovações desse setor de avanços em outras disciplinas científicas.

Em terceiro lugar, recursos privados são aplicados em universidades. Segundo Bond \& Glynn (p. 25), 5\% dos recursos do P\&D da indústria são alocados para universidades. Há uma extensa lista de programas apoiados pelo setor privado em diversas unidades do ensino superior norte-americano (p. 31).

Em quarto lugar, os testes clínicos dos novos medicamentos consomem grandes e crescentes somas do total do P\&D farmacêutico. Para Bond \& Glynn (p. 2528), os testes clínicos consumiriam cerca de $30 \%$ do total do P\&D do setor. A dimensão desses gastos é importante para explicar dificuldades que novas firmas biotecnógicas (NFBs) podem vir a encontrar para viabilizar financeiramente inovações até a fase de comercialização.

Em quinto lugar, a natureza da pesquisa farmacêutica apresenta mudanças: as doenças crônicas passaram a consumir $2 / 3$ (dois terços) dos investimentos em P\&D da indústria. Isso expressa por um lado, a sensibilidade da direção do progresso tecnológico do setor a mudanças na natureza da demanda e, por outro lado, o sentido da mudança da demanda como um reflexo de importantes mudanças epidemiológicas resultantes de uma expansão na expectativa de vida da população norte-americana (e dos países desenvolvidos em geral).

Finalmente, é interessante desagregar os investimentos especificamente acadêmicos no setor, a partir de dados do National Science Foundation (1998): as Ciências Biológicas consumiram US\$ 3,8 bilhões $(17,4 \%$ do total dos gastos acadêmicos) e as Ciências Médicas US\$ 6,1 bilhões (27,4\%). O National Institutes of Health (NIH) destaca-se como a mais importante fonte de recursos federais para pesquisa acadêmica (47,2\% do total dos recursos em 1991, segundo Rosenberg \& Nelson, 1994).

\section{I.4. Diferentes Padrões de Progresso Tecnológico no Setor}

A diversidade de padrões de avanço tecnológico no setor é outra importante particularidade. $\mathrm{Na}$ verdade, a amplitude de produtos e serviços envolvidos (desde a produção de seringas até tomógrafos computadorizados e proteínas geneticamente manipuladas) explica a pluralidade de padrões de progresso tecnológico.

\section{a) Biotecnologia}

Rosenberg \& Nelson (1994, p. 221) mencionam a visão dos executivos de P\&D sobre a pesquisa universitária como "fonte" de invenções. Os executivos explicavam isso pela novidade das tecnologias, e especulavam que com o tempo o papel da indústria deveria crescer em relação ao da universidade. Rosenberg $\&$ Nelson adicionam um comentário, destacando que o fato da tecnologia em si ter uma origem universitária é também pouco usual. 
Stern (1995) descreve a emergência da biotecnologia moderna, através da descrição do processo que levou à comercialização da insulina humana produzida a partir de engenharia genética. $\mathrm{O}$ autor destaca o grau em que departamentos universitários e empresas industriais estão organizados como insumos complementares de pesquisa. A história do desenvolvimento da insulina manipulada geneticamente fundamenta essa visão, pois ela envolve laboratórios de universidades como Harvard e Califórnia, o surgimento de um spin off como a Genetech e as suas relações com os laboratórios das universidades e uma grande empresa estabelecida no setor farmacêutico (Eli Lilly).

Arora \& Gambardella (1995) investigam a singular divisão do trabalho inovativo na biotecnologia, avaliando a interação entre três tipos de agentes: a) universidades, b) novas firmas biotecnológicas (NFBs) e c) grandes firmas estabelecidas da indústria farmacêutica.

Em linhas gerais, as universidades desempenham o já discutido papel de produção de novos conhecimentos científicos (desde descrição da estrutura de dupla hélice do DNA, com Watson \& Crick em 1953; até a técnica de desdobramento do gene - gene-splicing — com Cohen \& Boyer, em 1973). As NFBs apresentam vínculos fortes com o mundo acadêmico, sendo muitas vezes spin-offs da pesquisa universitária. Apresentam uma atmosfera universitária, mas diferenciam-se das universidades por perseguirem objetivos claramente definidos e de uso potencialmente comercial. Têm habilidade para sintetizar novas proteínas. São fracas para as fases de desenvolvimento, testes e comercialização. Por isso, muitas vezes as NFBs terminam vendendo seus produtos potenciais para grandes empresas. As grandes empresas estabelecidas do setor farmacêutico possuem alta capacidade de engenharia (know-how para levar um novo produto de laboratório a uma escala industrial), recursos financeiros para realizar os longos e custosos testes clínicos e uma estrutura de comercialização bem sofisticada. A estratégia das grandes empresas em relação às NFBs tem abarcado acordos de pesquisa, compra de participações minoritárias ou aquisição de NFBs.

\section{b) Indústria Farmacêutica}

Uma indústria intensiva em $P \& D$, com sofisticados laboratórios que podem empregar pesquisadores detentores de Prêmios Nobel ou capazes de produzir trabalhos posteriormente premiados, tem uma relação mais "convencional" (do tipo de indústrias de alta tecnologia) com as universidades. Elas dependem fortemente de pesquisas acadêmicas recentes. Mas têm importantes diferenças com o caso da biotecnologia. Rosenberg \& Nelson (1994, p. 221-222) mostram que os executivos do setor diferenciam claramente a origem de novas drogas não-biotecnológicas, pois essas teriam sido desenvolvidas na própria indústria. Porém, os entrevistados "também observaram que a pesquisa acadêmica havia esclarecido alguns tipos de reações bioquímicas que as companhias farmacêuticas poderiam investigar na busca por novas drogas, ou permitido às companhias realizarem uma avaliação mais efetiva de possíveis novos usos de drogas que já estavam testando" (p. 222). 
Essa posição é também sugerida por Arora \& Gambardella (1995, p. 189), que apontam que até o início dos anos oitenta a maioria das novas drogas provinha de investimentos sistemáticos em P\&D realizados pelas grandes empresas farmacêuticas. A emergência da biotecnologia moderna altera esse quadro, inicialmente estabelecendo um novo fluxo de informações entre as emergentes NFBs e a antiga indústria farmacêutica, além de ampliar a importância dos laços com a pesquisa acadêmica. Esse laços são fortes visto que as NFBs, muitas vezes, são "spin-offs" de departamentos de universidades.

A importância dos testes clínicos para a aprovação dos novos medicamentos é uma característica compartilhada com a indústria biotecnológica: como ressaltado anteriormente, os mecanismos de seleção de inovações são altamente regulados e nenhuma inovação chega às estruturas de assistência médica sem antes passar por esses testes e pelas instituições de regulação. Para esses testes, os centros acadêmicos médicos e hospitais são importantes.

\section{c) Indústria de Equipamentos Médicos}

A indústria de equipamentos médico-hospitalares caracteriza-se por um forte conteúdo interdisciplinar, além de depender intensamente de desenvolvimentos realizados em outras disciplinas científicas e em outras indústrias. O peso de spill-overs é enorme, descritos minuciosamente em estudos de casos de importantes inovações médicas. Os exemplos são diversos e muito interessantes (Gelijns \& Rosenberg, 1995). A aplicação da ressonância magnética na medicina dependeu de avanços dos físicos no estudo da estrutura do átomo, a ultra-sonografia foi filha da guerra submarina, a tomografia computadorizada foi conseqüência de avanços na computação e na matemática, o aparelho de litotripsia surgiu a partir de descobertas casuais da indústria aeronáutica.

O conjunto de relações com outros setores industriais não pode ser subestimado: não é casual que tantas inovações médicas provenham de empresas já estabelecidas em outros setores (GE, Siemens, HP etc). Boa parte das inovações médicas são excelentes exemplos de "economias de escopo" e estão relacionadas com diversificação de atividades de grandes empresas.

Porém, é necessário considerar que nenhuma dessas inovações médicas foi consequiência automática de avanços de outras disciplinas. Os desenvolvimentos das inovações em equipamentos médicos são processos que podem ser caracterizados como fortemente incrementais. Nesses processos o envolvimento de especialistas médicos é crucial: para a identificação da necessidade e da possibilidade de um novo equipamento, para a criação do primeiro protótipo de uma aplicação de tecnologias disponibilizadas por outras disciplinas e indústrias, e para aprimoramentos decisivos para o desenvolvimento do equipamento. O processo de inovação incremental ocorre não apenas nos laboratórios industriais, mas também na prática clínica.

As inovações na indústria de equipamentos médicos ressaltam a necessidade de uma ampla formação de quadros técnicos e científicos especializados para via- 
bilizar o aproveitamento de oportunidades tecnológicas geradas por uma multiplicidade de fontes. O papel das instituições de formação (no setor científico e no setor médico) é óbvio aqui. Além disso, o papel da pesquisa básica como formadora de mão-de-obra especializada para as atividades inovativas da indústria, como propõe Pavitt (1990) é particularmente visível nesse setor.

\section{d) Procedimentos Clínicos}

Um tipo de inovação importante para a prática médica, não necessariamente mediada por nenhuma indústria, mas resultando de uma interação direta entre universidades, centros acadêmicos médicos e do sistema de assistência médica. Como Gelijns (1990, p. 172) ressalta, novos procedimentos clínicos (e cirúrgicos) não passam por um sistema de regulação governamental (ao contrário de novos equipamentos e novos medicamentos), dependendo de avaliação da profissão médica e de auto-regulação.

Gelijns (1990, p. 182) comenta que há pouca informação disponível sobre investimentos em P\&D para novos procedimentos clínicos. As mudanças nos mecanismos de reembolso das despesas hospitalares parecem estar afetando esse mecanismo de financiamento. Inúmeros artigos e estudos têm avaliado, com preocupação, o efeito dessas mudanças sobre as pesquisas clínicas (National Science Foundation, 1998, p. 5-10).

\section{I.5. Uma Interação Produtor-Usuário Sui Generis: O Papel dos Hospitais e da Profissão Médica}

A literatura sobre inovações destaca o papel das interações produtor-usuário para o desenvolvimento e o aprimoramento de novas tecnologias (Lundvall, 1988).

Ao longo desta seção, foram apresentadas várias facetas do caráter especial dessas interações no setor saúde.

A prática clínica é importante para aperfeiçoar medicamentos e equipamentos médicos. A qualidade da formação médica, portanto, deve incidir sobre a contribuição que a profissão pode oferecer a essa interação crucial para o desenvolvimento tecnológico do setor.

Hicks \& Katz (1996) contribuem para destacar o papel científico de instituições fora do circuito acadêmico. Chegam a apresentar uma analogia entre a descoberta da supercondutividade por pesquisadores da IBM e a descoberta do papel da Helicobacter Pylori em problemas digestivos por um clínico (p. 303). Ou seja, a contribuição científica da prática médica não pode ser desprezada, e nesse ponto os hospitais têm participação ativa.

Dada essa interação singular, a forma de organização das atividades do setor pode afetar o volume e a qualidade dessa contribuição potencial. Tema que será explorado na próxima seção. 


\section{AS INTERAÇÕES ENTRE O SISTEMA DE INOVAÇÃO NO SETOR SAÚDE E OS SISTEMAS DE BEM-ESTAR SOCIAL}

O setor saúde possui uma característica distintiva de outros setores econômicos: ele está situado na interseção entre os sistemas de bem-estar social e os sistemas de inovação (Campos \& Albuquerque, 1999).

\section{II.1. As Influências Recíprocas entre Saúde e Crescimento Econômico}

Não é difícil compreender que crescimento econômico contribui para melhorias na saúde. É fácil estabelecer uma correlação entre melhorias na saúde em geral e renda per capita mais elevada, maior consumo de bens e serviços, melhor infra-estrutura de um país. De uma forma sintética e rudimentar, é possível afirmar que crescimento econômico causa melhorias na saúde.

A relação inversa (saúde causando crescimento econômico) não é difícil de ser pensada, pois melhorias na condição de saúde de um país devem repercutir sobre as fontes do crescimento econômico. Um exemplo importante é fornecido pelas pesquisas de Fogel (1994): 30\% do crescimento econômico da Grã-Bretanha, nos últimos duzentos anos, pode ser atribuído a melhorias em nutrição (p. 386).

O Relatório do Banco Mundial (World Bank, 1993) sistematiza alguns pontos onde melhorias em saúde influem sobre o crescimento econômico. Destacam-se os seguintes pontos: a) ganhos na produtividade do trabalho; b) melhor utilização de recursos naturais; c) benefícios que a educação pode trazer para próximas gerações; d) redução nos custos da assistência médica; e) influência de investimentos em saúde sobre a redução da pobreza.

Somando os efeitos, é apresentada a conclusão de que "melhoria nas condições de saúde deve conduzir à melhoria do desempenho econômico no âmbito nacional" (p. 23) e que "os dados indicam que melhores condições de saúde representam crescimento mais acelerado" (p. 25).

Porém, as relações entre crescimento econômico e saúde são mais complexas.

Em primeiro lugar, Amartya Sen (1992) propõe uma importante qualificação das relações entre crescimento econômico e melhorias na saúde. Sen (1992, p. 1306) descreve suas pesquisas como tendo avaliado "por quê e como o crescimento econômico tem sido mais bem sucedido em certos casos do que em outros na redução da mortalidade e na expansão da longevidade". Sen (p. 1310) explicita que a questão não é nem que o crescimento econômico seja pouco importante na redução da mortalidade nem que ele seja uma solução completa: outras questões, como serviços médicos podem ser importantes, "requerendo que se avance para além das análises de crescimento e distribuição baseadas na renda para a questão particular de medidas de saúde pública e de acesso à assistência médica".

Em segundo lugar, em geral a expectativa de vida (uma espécie de indicador síntese de melhorias em condições de saúde) cresce com a renda per capita. Porém, ao longo do século XX há um desvio ascendente nessa relação. Um exemplo, descrito no Relatório facilita a compreensão desse "desvio ascendente". Em 1900, a 
expectativa de vida nos Estados Unidos era de 49 anos, correspondendo a uma renda per capita de US\$4.800,00. Essa renda per capita, em 1990 (valores devidamente ajustados) estaria associada a uma expectativa de vida de 71 anos. A relação entre renda e expectativa de vida pode ser estabelecida, mas uma ascensão deve ser identificada: ao longo deste século, curvas diferentes descrevem a correlação entre renda e expectativa de vida para períodos diferentes.

Esse "desvio ascendente" sugere que há outros fatores operando entre o crescimento econômico e a saúde. Como o Relatório do Banco Mundial (1993, pp. 3738) sintetiza, as "lições do passado" sobre a queda da mortalidade indicam que três fatores contribuem para explicar as quedas de mortalidade: a) aumento da renda per capita; b) avanços na tecnologia médica; c) o desenvolvimento da saúde pública e disseminação de conhecimentos.

Enfim, a interação entre crescimento econômico e saúde é não-automática, mas multidimensional, recíproca, mutuamente determinante e de efeitos crescentes, ascendente. Nessa interação desempenham um papel de intermediação crucial a intervenção do desenvolvimento de tecnologias médicas e da melhorias na saúde pública assim como a forma de organização dos serviços e a abrangência da cobertura.

\section{II.2. Uma Visão Dinâmica sobre a Relação entre o Desenvolvimento Científico-Tecnológico e Custos no Setor Saúde}

A dinâmica de inovação tecnológica no setor saúde tem sido considerada como uma das razões para o crescimento dos gastos do setor. É verdade que um hospital moderno típico consome pesados investimentos na compra de equipamentos médicos, sofisticados e caros. Porém, essa associação direta entre progresso tecnológico e altos gastos deve ser avaliada com mais cautela (Barr, 1992).

Weisbrod (1991) aponta evidências problematizando que as inovações tecnológicas sejam exclusivamente encarecedoras da assistência médica (p. 531). Documento da OCDE deixa em aberto essa questão, apresentando dúvidas "se as novas tecnologias são parte do problema, parte da solução, ou as duas coisas" (OECD, 1998, p. 3).

Para avaliar esse ponto, Weisbrod compara vacinas e transplantes, seus custos, repercussões e respectivas demandas por inovações. Para explicitar sua posição, utiliza como ponto de partida a elaboração do biólogo Lewis Thomas (de 1975), que distingue três estágios de desenvolvimento tecnológico na medicina:

No nível mais baixo, "não-tecnologia" (nontechnology), onde os vínculos entre o paciente e a doença são fracamente compreendidos. Pouco pode ser feito pelo paciente, apenas a hospitalização e serviços de enfermaria, com pequena esperança de recuperação (câncer não-tratável, artrite reumatóide severa, esclerose múltipla, cirrose avançada);

Um pouco acima, "tecnologias intermediárias" (halfway technology), que incluiria lidar com a doença e com os seus efeitos incapacitantes depois de estabelecida. Tratam-se de tecnologias que ajustam o paciente à doença e adiam a morte 
(implantação de órgãos artificiais e transplante, tratamento de câncer por cirurgia, radiação e quimioterapia).

"Alta tecnologia”, exemplificada por imunização, antibióticos, prevenção de desordens nutricionais, trata de doenças cujos mecanismos são conhecidos e cujo tratamento/prevenção é viável.

Weisbrod (1991, p. 533) sugere tornar esse esquema dinâmico: historicamente o conhecimento passa do primeiro para o segundo e a seguir para o terceiro tipo de tecnologia. Dessa sugestão, deriva que a função de custo associada a esse processo dinâmico tenha a forma de um U-invertido: no caso de não-tecnologia, há pouco a se fazer e os gastos são baixos, o ponto mais caro seria nas tecnologias intermediárias, voltando a cair no caso do terceiro estágio ("alta tecnologia”). Como exemplo Weisbrod usa a evolução da pólio: a) no início (duas gerações atrás) suas vítimas morriam rapidamente como resultado da paralisia; b) depois houve o desenvolvimento de fase de "tecnologia intermediária", com o surgimento do pulmão artificial (iron lung), que prolongava a vida a custos substanciais; c) finalmente, as vacinas (Sabin e Salk) da fase de "alta tecnologia" reduziram dramaticamente os custos associados à pólio. (pp. 533-534). ${ }^{5}$

Aplicando o conceito de "trajetórias tecnológicas"(Dosi, 1988) ao caso da saúde, poderiam ser sugeridas as seguintes:

- técnicas de tratamento e diagnóstico menos invasivas: um exemplo é o de-senvolvimento da endoscopia, ultra-sonografia, técnicas de imagens cardíacas etc; outro exemplo seria a invenção do aparelho de litotripsia (que substitui o uso de técnicas cirúrgicas na remoção de cálculos renais);

- desenvolvimento de medicamentos mais eficazes, o que significa substituiçãode cirurgias e/ou de longos períodos de internação hospitalar;

- desenvolvimento de vacinas, que são conseqüência de maior conhecimentosobre processos do corpo humano, evitando gastos com o tratamento de doenças que se tornam passíveis de prevenção efetiva;

- crescente reconhecimento da importância de hábitos, condições de vida e tra-balho, regimes alimentares sobre as condições de saúde: a contínua geração de informações que devem ser propagadas via educação e meios de comunicação pode ser um poderoso redutor de gastos (exemplo: redução do número de fumantes e o seu efeito sobre gastos hospitalares para tratamento de doenças associadas ao fumo);

- mesmo em termos de equipamentos médicos, é possível supor que ela acom-panha a tendência geral de miniaturização, ampliação de sua capacidade e

\footnotetext{
${ }^{5}$ Um questionamento desse esquema pode ser apresentado, restringindo a sua validade. Ele seria válido para doenças transmissíveis e para doenças crônicas que atingem pessoas abaixo dos 65 anos de idade. Entretanto, na medida que a expectativa de vida fosse sendo ampliada, o custo para cada novo ano ganho teria uma tendência ascendente.
} 
barateamento que ocorre, por exemplo, nas indústrias de computadores e telecomunicações.

Ou seja, nas cinco "trajetórias tecnológicas" da saúde, descritas acima, é possível identificar claramente elementos redutores de custos. A conclusão, mesmo que provisória e sujeita a importantes qualificações, é a de que a continuidade e a aceleração do progresso tecnológico no setor exercerão impactos sobre a prática médica no sentido de barateá-la.

Há dois problemas iniciais acerca dessa visão. Em primeiro lugar, certos avanços na área de medicamentos podem levar à viabilização de novos tipos de cirurgia e procedimentos caros: Weisbrod exemplifica com o impulso recebido pelas cirurgias de transplante após o desenvolvimento de drogas imunosupressoras (pp. 539540). Em segundo lugar, é importante observar os fatores que governam a oferta de inovações médicas, como insistem Gelijns \& Rosenberg (1995): um bom exemplo dessa limitação é oferecida pela biotecnologia cujas promessas são expressivas, mas que até o momento "a eficácia clínica não tinha sido demonstrada de forma definitiva em nenhuma terapia genética" (OECD, 1998, p. 28). Talvez "a terapia genética demore mais do que o previsto para alcançar os pacientes” (p. 28).

Limitações no desenvolvimento científico tornam a oferta de inovações praticamente inelástica por alguns períodos de tempo (Rosenberg, 1976).

\section{II.3. Estrutura de Incentivos, Direção e Intensidade do Progresso Tecnológico no Setor Saúde}

Definidas as linhas básicas de evolução científica e tecnológica no setor, os constrangimentos oferecidos pelo estágio da ciência e as diversas possibilidades e "trajetórias" existentes, é necessário avaliar como a demanda e a estrutura de incentivos derivada da organização assumida por ela podem afetar a direção e a intensidade do progresso tecnológico no setor.

Essa sensibilidade do sentido do progresso tecnológico em relação à estrutura de incentivos é importante. Halm \& Gelijns (1991), comentando um conjunto de trabalhos sobre esse tema, consideram que "se torna evidente que o ponto crítico aqui não é a tecnologia médica per se, mas uma combinação de incentivos econômicos, profissionais e sociais no sistema de atenção à saúde que tende a diminuir a preocupação com os custos nas decisões de cuidados médicos” (p. 1).

Weisbrod (1991) articula a forma de organização dos serviços de saúde nos Estados Unidos com os incentivos e sinais que esse sistema produzia para as atividades de pesquisa das indústrias do setor. Weisbrod sugere que o desenvolvimento recente de "tecnologias intermediárias" (tecnologias caras, de caráter paliativo como transplantes, hemodiálise etc) teria sido "implicitamente encorajado pelo esquema de reembolso adotado pelo sistema de seguro que dominou os hospitais e a assistência médica até recentemente, porque havia pouco ou nenhum incentivo para os fornecedores de serviços evitarem tecnologias caras que fossem apenas marginalmente efetivas" (1991, p. 534). 


\section{II.4. Diferentes Regimes de Regulação: Os Arranjos Governo-Academia-Indústria no Reino Unido e na França}

Desdobrando a discussão para questões relacionadas à estrutura industrial, é útil uma comparação entre o sistema setorial de inovação no Reino Unido e na França, estudada por Thomas (1994). Essa comparação demonstra que não é importante apenas o volume dos gastos públicos com ciência e tecnologia, mas também a forma de articulação entre os vários setores em termos de execução e regulação das atividades do setor saúde.

O sistema de inovação britânica conta com uma base científica extremamente complexa, capaz e eficiente. O financiamento público à pesquisa é elevado mas é complementado por um envolvimento também elevado por parte do setor produtivo. Já no caso francês, apesar do elevado montante de recursos públicos alocado ao financiamento da pesquisa, observa-se um envolvimento pobre do setor produtivo (Thomas, 1994).

As diferenças na constituição do sistema de inovação ligado ao setor de saúde em ambos os países passam por dois tipos de regulação: a de segurança da saúde pública e a de preços. No que se refere à regulação da segurança de saúde pública, no Reino Unido, inicialmente, ela foi realizada pelo Comittee on Safety of Drugs (CSD), uma organização "quase governamental”, composta de especialistas acadêmicos, independentes e voluntariamente organizados, e apoiados pela indústria. $\mathrm{O}$ CSD foi substituído em 1971 pelo Comittee on Safety of Medicines (CSM), uma organização governamental formal. Desde o início a regulação se preocupou com a medicina acadêmica formal, particularmente no que se refere a testes clínicos controlados para se demonstrar a segurança e eficácia das novas drogas. Documentação extensa e altos padrões acadêmicos eram necessários para todos os pedidos. Deve-se reconhecer tanto a pronunciada cooperação do CSD/CSM com o setor industrial quanto a dependência do CSD/CSM com relação à indústria e à academia para a formulação dos procedimentos técnicos e padrões pelos quais os testes seriam avaliados. Tal colaboração resultou numa coletivização elitista do processo de tomada de decisões sobre níveis de segurança e eficácia de maneira a que altos padrões eram colocados a todos os participantes do mercado, quer grandes ou pequenas empresas, quer empresas locais ou multinacionais (Davies 1967).

A regulação na França evoluiu de maneira diferente. O registro de novas drogas começou em 1941, sob uma legislação protecionista que requeria que produtos vendidos na França fossem produzidos por laboratórios franceses. Em 1959, a legislação foi alterada prevendo proteção patentária, permitindo o controle de laboratórios por empresas estrangeiras e estabelecendo que a segurança e qualidade de novas drogas fosse demonstrada de maneira a satisfazer um expert agrée. Este expert tinha que ser escolhido e pago pela empresa que tivesse desenvolvido o novo medicamento, a partir de uma lista de acadêmicos compilada pelo Ministério da Saúde. Apesar de mudanças, duas diferenças importantes permanecem com relação ao sistema britânico. Em primeiro lugar, decisões sobre o acesso ao mercado local são feitas, no caso francês por indivíduos, pagos pelas empresas ao invés de 
coletivamente por comitês de especialistas independentes como no caso britânico. Em segundo lugar, o cerne da decisão sobre a entrada ou não no mercado, no caso francês, refere-se a segurança e qualidade baseadas em métodos de avaliação tradicionais por médicos individuais. No caso britânico, os testes formais de drogas consideram adicionalmente a eficácia da droga demonstrada por testes clínicos controlados. Evidentemente eficácia é muito mais difícil, custosa e demorada de se demonstrar do que segurança e é exatamente a demonstração de eficácia que distingue o sistema britânico do francês. Como resultado o número de drogas introduzidas no mercado britânico tem sido significativamente menor no Reino Unido do que na França. Também, o número de firmas inovativas (se medido por novas drogas introduzidas no mercado local) era muito maior na França do que no Reino Unido e elas eram menores. Porém a qualidade da inovação no Reino Unido é muito melhor do que na França, o que pode ser medido pela participação relativa das empresas dos dois países no comércio internacional - em 1985 as empresas britânicas tinham uma external share (definida como as vendas das empresas daquele país como uma porcentagem das vendas de produtos farmacêuticos em todos os mercados estrangeiros) de $5,8 \%$, enquanto o indicador correspondente para as empresas francesas era de 1,0\% (Thomas 1994).

O outro ponto importante refere-se à regulação de preços para novas drogas. $\mathrm{Na}$ maior parte dos países desenvolvidos o governo é um grande comprador de drogas. O problema de se estabelecer preços para as compras governamentais é resolvido de maneira bem diferente em ambos os países e isso têm muito a ver com os efeitos nos sistemas de inovação. No Reino Unido a regulação tem sido feita a partir de uma cooperação voluntária entre indústria e o Ministério da Saúde através inicialmente do Voluntary Price Regulation Scheme (VPRS) e após 1978 do Pharmaceutical Price Regulation Scheme (PPRS). Tecnicamente os preços não são negociados com relação a drogas/produtos individuais, mas nos lucros de cada empresa como um todo, calculado como a taxa de retorno sobre um nível acordado de investimento de capital. Os retornos das empresas podem ser aumentados sob tal esquema ou através do aumento da taxa de retorno ou aumentando o nível de investimentos. A TIR é negociada diretamente e é maior para empresas orientadas para exportação. O resultado direto é uma forte discriminação contra empresas que queiram lucrar do mercado britânico sem investir em esforços inovativos locais. Firmas fracas, imitativas serão penalizadas duplamente pois terão baixos níveis de investimentos e baixa taxa de retorno; portanto independentemente do esquema de regulação o PPRS levou a uma concentração favorecendo as empresas grandes e fortes. Da mesma maneira firmas estrangeiras que querem apenas vender no mercado local são penalizadas e terão os seus preços controlados mais fortemente.

$\mathrm{Na}$ França os preços são determinados por produtos/drogas, negociados com o Ministério da Fazenda. Até 1968 a fórmula era um mark-up sobre custos de produção apenas (cadre des prix). Como resultado os investimentos em P\&D são desencorajados; pelo contrário altos custos de produção eram encorajados, como montagem manual, uso de compostos etc. Mais ainda, como os preços não eram ajustados pela inflação as firmas eram compelidas a fazer "pseudo-novidades". 
Após 1968 tal formula é modificada permitindo uma definição mais ampla de custos; porém o controle de preços foi continuando com seu viés anti-inovação. Assim, a política de regulação de preços age como estímulo à busca de inovações, o que se traduz num aumento de gastos em P\&D por parte do setor empresarial, inclusive na academia. No caso francês, a regulação de preços não estimula o aumento de gastos privados em P\&D e isso se traduz num baixo envolvimento do setor industrial com a pesquisa acadêmica.

\section{CONCLUSÃO}

Sintetizando as discussões deste trabalho, cinco pontos expressam as idéias principais encontradas na literatura e nos dados aqui investigados:

Um sistema nacional de inovação pode ser decomposto em componentes setoriais. O setor saúde é um componente importante, dado o seu papel de interseção entre o sistema de inovação e o sistema de bem-estar social.

O sistema de inovação do setor saúde é fortemente baseado na ciência. $\mathrm{O}$ peso das universidades e das instituições de pesquisa nos fluxos de informação tecnológico é expressivo.

O peso do setor universitário e científico no sistema de inovação de saúde indica a relevância dos investimentos públicos na área.

As especificidades da atenção médica como atividade econômica são consideráveis, determinando um papel importante para instituições no setor.

Em função da interação entre o sistema setorial de inovação no setor saúde e o sistema de bem-estar social, o papel da regulação das atividades do setor tem profundas influências sobre a direção do progresso tecnológico e sobre os arranjos institucionais, afetando fortemente o desempenho econômico, industrial e social do conjunto da área de saúde.

Este artigo, ao sintetizar a avaliação dos sistemas setoriais de inovação da saúde, busca contribuir para uma investigação das particularidades do caso brasileiro. A principal lição dessa discussão introdutória para o caso brasileiro está na forte sensibilidade do setor saúde aos diferentes arranjos institucionais. Incentivos importam e afetam a forma como são focalizadas trajetórias de progresso tecnológico.

Tanto pelo lado de das universidades, como pelo lado de políticas industriais e de regulação econômica, o papel da atuação do setor público é decisivo na moldagem da capacidade inovativa do setor saúde. Talvez, mais do que em qualquer outro setor econômico, a inter-relação entre as instituições públicas e privadas na constituição da dinâmica inovativa envolve uma forte participação (direta e indireta) do setor público. A implementação de políticas de catching up setorial deve partir de uma reflexão sobre essa combinação. Tema para posterior discussão. 


\section{REFERÊNCIAS BIBLIOGRÁFICAS}

ALBUQUERQUE, E. (1999) "National Systems of Innovation and Non-OECD Countries: Notes About a Tentative Typology". Revista de Economia Política, 19 (4).

ARORA, A. e GAMBARDELlA, A. (1995) "The Division of Innovative Labor in Biotechnology". In ROSENBERG, N., GELIJNS, A. e DAWKINS, H. (1995).

BARR, N. (1992) "Economic Theory and the Welfare State: a Survey and Interpretation". Journal of Economic Literature, 30 (2): 741-803.

BOND, E.; GLYNN, S. (1995) "Recent trends in support for biomedical research and development". In ROSENBERG, N., GELIJNS, A. e DAWKINS, H. (1995)

BRESCHI, S. e MALERBA, F. (1997) "Sectoral Innovation Systems: Technological Regimes, Schumpeterian Dynamics, and Spatial Boundaries”. In: EDQUIST, C. (ed.). Systems of Innovation: Technologies, Institutions and Organizations. London: Pinter,: 130-156.

CAMPOS, F. E. e ALBUQUERQUE, E. (1999) “As Especificidades do Trabalho no Setor Saúde”. Revista de Economia Contemporânea, 3 (2): 97-123.

CORDEIRO, H. (1980) A Indústria da Saúde no Brasil. Rio de Janeiro: Graal.

DAVIES, W. (1967) The Pharmaceutical Industry: a Personal Study. Pergamon Press: Oxford.

DOSI, G. (1988) “Sources, Procedures and Microeconomic Effects of Innovation". Journal of Economic Literature, 26 (3): 1120-1171.

FOGEL, R. (1994) "Economic Growth, Population Theory, and Physiology: the Bearing of Long-term Processes on the Making of Economic Policy". American Economic Review, 84 (3).

FREEMAN, C. (1995) “The 'National System of Innovation' in Historical Perspective”. Cambridge Journal of Economics, 19 (1).

GELIJNS, A. (1990) "Comparing the Development of Drugs, Devices and Clinical Procedures”. In: GELIJNS, A. Modern Methods of Clinical Investigation. (Medical innovation at the crossroads, v. 1). Washington: National Academy.

GELIJNS, A. e ROSENBERG, N. (1995) “The Changing Nature of Medical Technology Development". In ROSENBERG, N., GELIJNS, A. e DAWKINS, H. (1995).

HALM, E. e GELINJS, A. (1991) "An Introduction to the Changing Economics of Technological Innovation in Medicine". In: GELIJNS, A. e HALM, E. (eds.). The Changing Economics of Medical Technology (Medical innovation at the crossroads, v. 2). Washington: National Academy.

HICKS, D. e KATZ, J. (1996) "Hospitals: the Hidden Research System”. Science and Public Policy, 23 (5): 297-304, October.

KLEVORICK, A., LEVIN, R., NELSON, R. e WINTER, S (1995). "On the Sources and Significance of Inter-industry Differences in Technological Opportunities”. Research Policy, 24: 185-205.

LUNDVALL, B-A (ed.) (1992) National Systems of Innovation: Towards a Theory of Innovation and Interactive Learning. London: Pinter.

MAINSFIELD, E. (1991) “Academic Research and Industrial Innovation”. Research Policy, 20: 1-12.

MURRAY, C. J. (ed.); LOPEZ, A. (1996) The Global Burden of Disease: a Comprehensive Assessment of Mortality and Disability From Diseases, Injuries, and Risk Factors in 1990 and Projected. Harvard: Harvard School of Public Health.

MYTELKA, L. e DELAPIERRE, M. (1999) "Strategic Partnerships, Knowledge-based Oligopolies and the State”. In: CLUTER, C., HAUFLER V. e PORTER, T. (eds) Private Authority and International Affairs. Binghamton: Suny University: 129-149.

NARIN, F., HAMILTON, K. S. e OLIVASTRO, D. (1997) “The Increasing Linkage Between U.S. Technology and Public Science”. Research Policy, v. 26, (3): 317-330.

NATIONAL SCIENCE FOUNDATION (1998). Science and Engineering Indicators 1998. Washington: National Science Foundation. (http://www.nsf.gov)

NELSON, R. (1995) "The Intertwining of Public and Proprietary in Medical Technology". In ROSENBERG, N., GELIJNS, A. e DAWKINS, H. (1995).

NELSON, R. e WINTER, S. (1982) An Evolutionary Theory of Economic Change. Cambridge, Mass; London: The Belknap Press of Harvard University Press. 
OECD (1998) Economic Aspects of Biotechnologies Related to Human Health (Part I). (http:// www. oecd.org).

PAVITT, K. (1991) “What Makes Basic Research Economically Useful?” Research Policy, 20 (2): 109119.

ROSENBERG, N. (1976) Perspectives on Technology. Cambridge: Cambridge University.

ROSENBERG, N., GELIJNS, A. e DAWKINS, H. (1995) Sources of Medical Technology: Universities and Industry (Medical innovation at the crossroads, v. 5). Washington: National Academy.

ROSENBERG, N. e NELSON, R. (1994) “American Universities and Technical Advance in Industry". In: NELSON, R. The Sources of Economic Growth. Cambridge, Mass: Harvard University, 1996. SEN, A (1992) "Life and Death in China: a Reply". World Development, 20 (9): 1305-1312.

STERN, S. (1995) "Incentives and focus in university and industrial research: the case of synthetic insulin”. In ROSENBERG, N., GELIJNS, A. e DAWKINS, H. (1995).

THOMAS, L.G. (1994) "Implicit Industrial Policy: the Triumph of Britain over France in Global Pharmaceuticals". Industrial and Corporate Change, 3 (2).

WEISBROD, B. (1991) "The Health Care Quadrilemma: an Essay on Technological Change, Insurance, Quality of Care, and Cost Containment”. Journal of Economic Literature, 29 (2): 523-552.

WORLD BANK (1993) World Development Report 1993: Investing in Health. Oxford: Oxford University. 